\title{
Is There a Stobbs Factor in Atomic-Resolution STEM-EELS Mapping?
}

\author{
Huolin L. Xin, ${ }^{*}$ Christian Dwyer ${ }^{* *}$, David A. Muller, ${ }^{* * *}$ \\ * Department of Physics, Cornell University, Ithaca, NY 14853 \\ ** Monash Centre for Electron Microscopy, Monash University, Clayton 3800 Vic, Australia \\ ***School of Applied and Engineering Physics \& Kavli Institute, Cornell Univ., Ithaca, NY 14853
}

As noted by Crewe in his classic paper introducing the field-emission STEM: "it seemed that the most promising method for obtaining adequate contrast was by the use of energy-loss mechanisms." [1]. Here we consider the question of contrast in STEM-EELS maps. Recent work has convincingly argued that the Stobbs factor-disagreement in contrast between simulated and experimental atomic-resolution images - in HAADF-STEM imaging can be accounted for by including the incoherent source size in simulation [2]. However, less progress has been made for atomicresolution STEM-EELS mapping. This is hindered by two main issues. First, the EELS signal is several orders of magnitude weaker than that of HAADF, requiring a much more stable instrument. Second, the computation time required by an inelastic double-channeling simulation is several orders of magnitude longer than that required by a HAADF multislice simulation [3]. To shorten the computation time, a single-channeling model which neglects the subsequent multiple elastic scattering of the inelastically-scattered electrons is often used [4]. However, the failure of Blochwave simulations in moderate-thickness HAADF-STEM alerts us that the channeling of the inelastic signal is likely to substantially affect both the EELS-map contrast and the absolute signal there.

Here we report the first atomic-resolution STEM-EELS mapping with both the ADF and the EELS signals normalized to the incident beam (Fig 1), enabling a quantitative analysis of crosssections and contrast. This allows us to explore the interplay between elastic and inelastic contrast, determine the limits of the single-channeling approximation and demonstrate that the Stobbs factor for STEM-EELS mapping can be accounted for (Fig 2b) by the source-size determined from ADFSTEM (Fig 2a). We spectroscopically imaged $\mathrm{DyScO}_{3}$ (DSO) at a series of thicknesses ranging from $20 \mathrm{~nm}$ to $90 \mathrm{~nm}\left(\alpha_{\max } \approx 32 \mathrm{mrad}, \beta_{\max } \approx 80 \mathrm{mrad}, \mathrm{ADF} \approx 98-295 \mathrm{mrad}\right)$. The incoherent source size needs to be modeled as a Gaussian convolved with a truncated Lorentzian [5] in order to simultaneously match the ADF contrast of the Dy columns, the Sc columns and the background level (Fig. 2a). This is most likely due to the probe tail from residual geometric aberrations, chromatic aberration and instabilities during data acquisition. Using the EELS spectra obtained, we can map out Dy from the $N_{4,5}$ edge at $\sim 150 \mathrm{eV}$ and $M_{4,5}$ at $\sim 1300 \mathrm{eV}$, and Sc from the $L_{2,3}$ edge at $\sim 400 \mathrm{eV}$. As shown in Fig. 1, the contrast of the experimental maps agrees well with that of the double-channeling simulations convolved with the ADF-determined source size. It is worth noting that, however, a donut structure develops with thickness on the Dy columns when the integration windows moves onto the fano resonance peak of Dy- $N_{4,5}$. The final state on the resonance has a much more delocalized overlap with the $3 d$ initial states than that of the off-resonance continuum states. Therefore, the on-resonance map started with a relatively weak contrast. With the beam directly on the Dy columns, elastic and thermal diffuse scattering quickly depletes the beam on column, producing the donut structure. [6]

References

[1] A. V. Crewe, Science, 154729 (1966).

[2] J. M. LeBeau et al., Physical Review Letters 100, 206101 (2008).

[3] C. Dwyer, S. D. Findlay, and L. J. Allen, Physical Review B 77, 184107 (2008).

[4] M. Bosman et al., Physical Review Letters 99, 086102 (2007). 
[5] C. Dwyer, R. Erni, and J. Etheridge, Ultramicroscopy 110, 952 (2010).

[6] Funded by the Energy Materials Center at Cornell, an Energy Frontier Research Center (DOE \#DE-SC0001086). C.D. acknowledges financial support from the Australian Research Council (DP110104734)

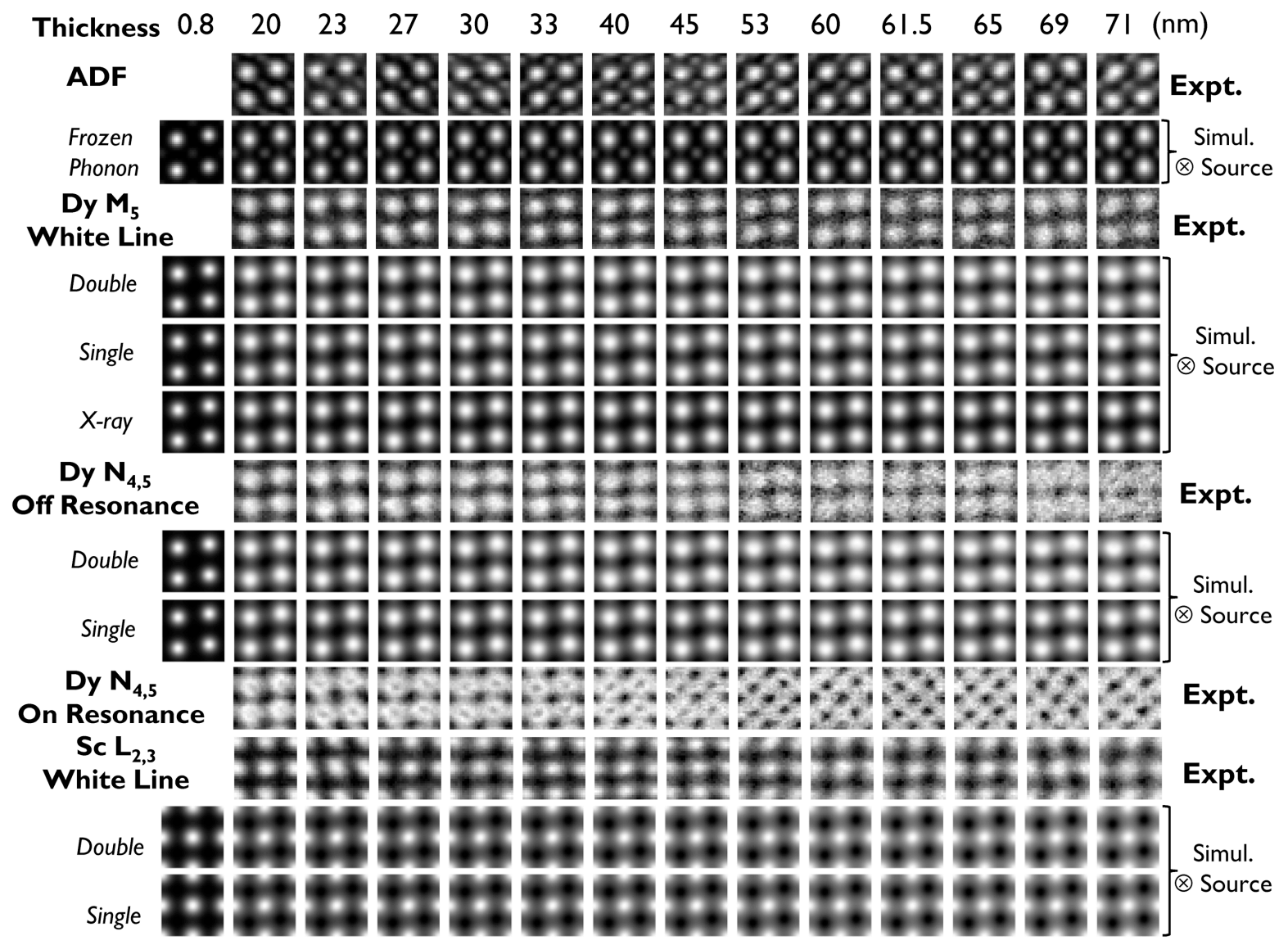

Fig. 1. Comparison of the EELS maps and simultaneously-acquired ADF images with simulations. The simulated images are convolved with the source size determined in Fig. 2a. Each experimental map is a position averaged result from a 10x10-unit cell EELS map and each image is on its own gray scale. (Dy $N_{4,5}$ On Resonance: $4 \mathrm{eV}$ window around the resonance at $\sim 164 \mathrm{eV}$; Dy $N_{4,5}$ Off Resonance: $200-230 \mathrm{eV}$ )

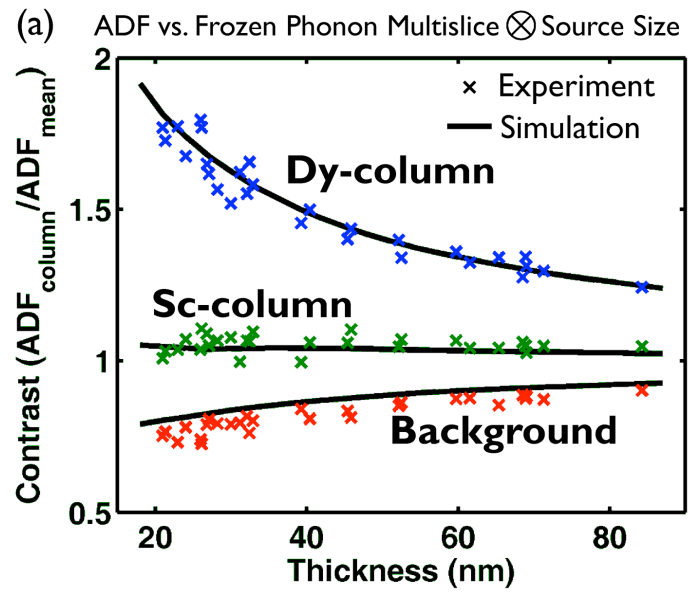

(b) EELS vs. Double-Channeling $\otimes$ Source Size

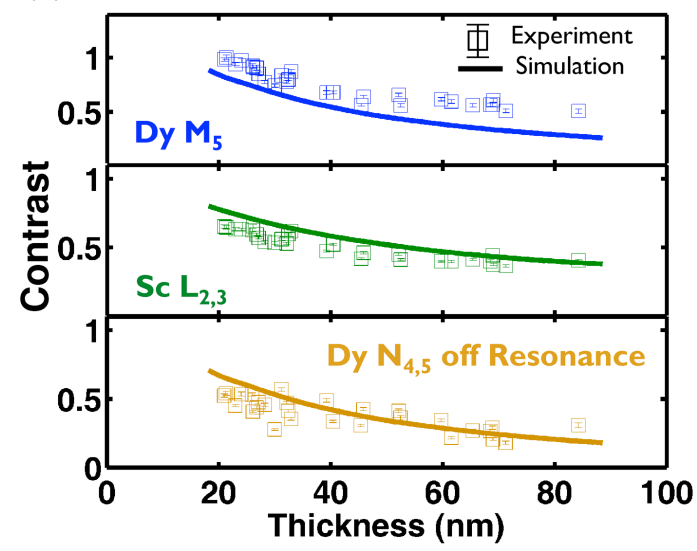

Fig. 2. (a) Quantitative match between the experimental contrast of the simultaneously-acquired ADF images with the frozen-phonon multislice simulations. The source size is determined to be a $0.81 \AA$-FWHM Gaussian convolved with a $0.15 \AA$-FWHM Lorentzian. (b) Comparison of the contrast in the EELS maps with the double-channeling simulation. The double-channeling simulations were convolved with the source size independently determined by the ADF-STEM analysis in (2a). 